\title{
Multimodal Analysis of Calcium Carbonate Polymorphs in Biological Systems
}

Ute Schmidt $^{1}$, Wei Liu ${ }^{2}$ and David Steinmetz ${ }^{1}$

${ }^{1}$ WITec GmbH, Ulm, Baden-Wurttemberg, Germany, ${ }^{2}$ WITec Instruments Corp., Knoxville, Tennessee, United States

Calcium carbonate $\left(\mathrm{CaCO}_{3}\right)$ is one of the most widely distributed compounds on Earth, often in the form of water insoluble calcite in sedimentary rocks. Other mineral forms of $\mathrm{CaCO}_{3}$ are aragonite and vaterite, which are found in rocks, but also as biogenic materials in sea shells and pearls. Land dwelling crustaceans form their protective shells from calcite, which they need to recycle by producing amorphous calcium carbonate due to the lack of enough calcium in non-aquatic environment $[1,2]$. Due to their different crystallographic structures (calcite - rhombohedral, aragonite - orthorhombic, and vaterite - hexagonal unit cell), the polymorphs of $\mathrm{CaCO}_{3}$ can be distinguished by Raman spectroscopy (Fig. 1a). Furthermore, the distribution of various forms of $\mathrm{CaCO}_{3}$ in the cuticle of a porcellio scaber can be visualized using confocal Raman imaging (Fig. 1b, c). The cuticle consists of an organic matrix composed of chitin-protein fibers associated with various amounts of crystalline calcite and amorphous calcium carbonate. Further structural details can be obtained using AFM imaging (Fig. 2d).

The coexistence of aragonite and vaterite can be demonstrated with the example of a milky pearl [3]. Pearls are produced by a natural biomineralization process controlled by organics and usually consists of aragonite. The structure is comprised of stacks of hexagonal platelets along the c-axis of the aragonite crystal, creating a pearlescent surface. Pearls occasionally reveal a milky surface as a result of a biomineralization defect. Such defects can be analyzed using high resolution SEM combined with chemical sensitive Raman imaging (RISE). Fig 2a,b show low vacuum SEM images of the structure of vaterite and aragonite of the analyzed pearl. Fig. 2c shows a RISE image of the border between pearlescent and opaque areas of the milky pearl. The high-resolution SEM image reveals the hexagonal platelets of aragonite on one side of the image and elongated features on the other half of the image corresponding to the needle-like structure of vaterite. These two structures are confirmed by the Raman spectra acquired from the corresponding areas. Furthermore, the anisotropy of the aragonite platelets of the pearl is detected by Raman imaging and highlighted in the Ca-lattice vibrations of the Raman spectrum (Fig. 2d). Such anisotropies are reported for biogenic aragonite using different experimental methods [4].

The aim of this presentation is to reveal unique structures of $\mathrm{CaCO}_{3}$ polymorphs grown by organisms and their properties using multimodal imaging such as Raman-AFM or Raman-SEM, integrated within one instrument. 
(a)
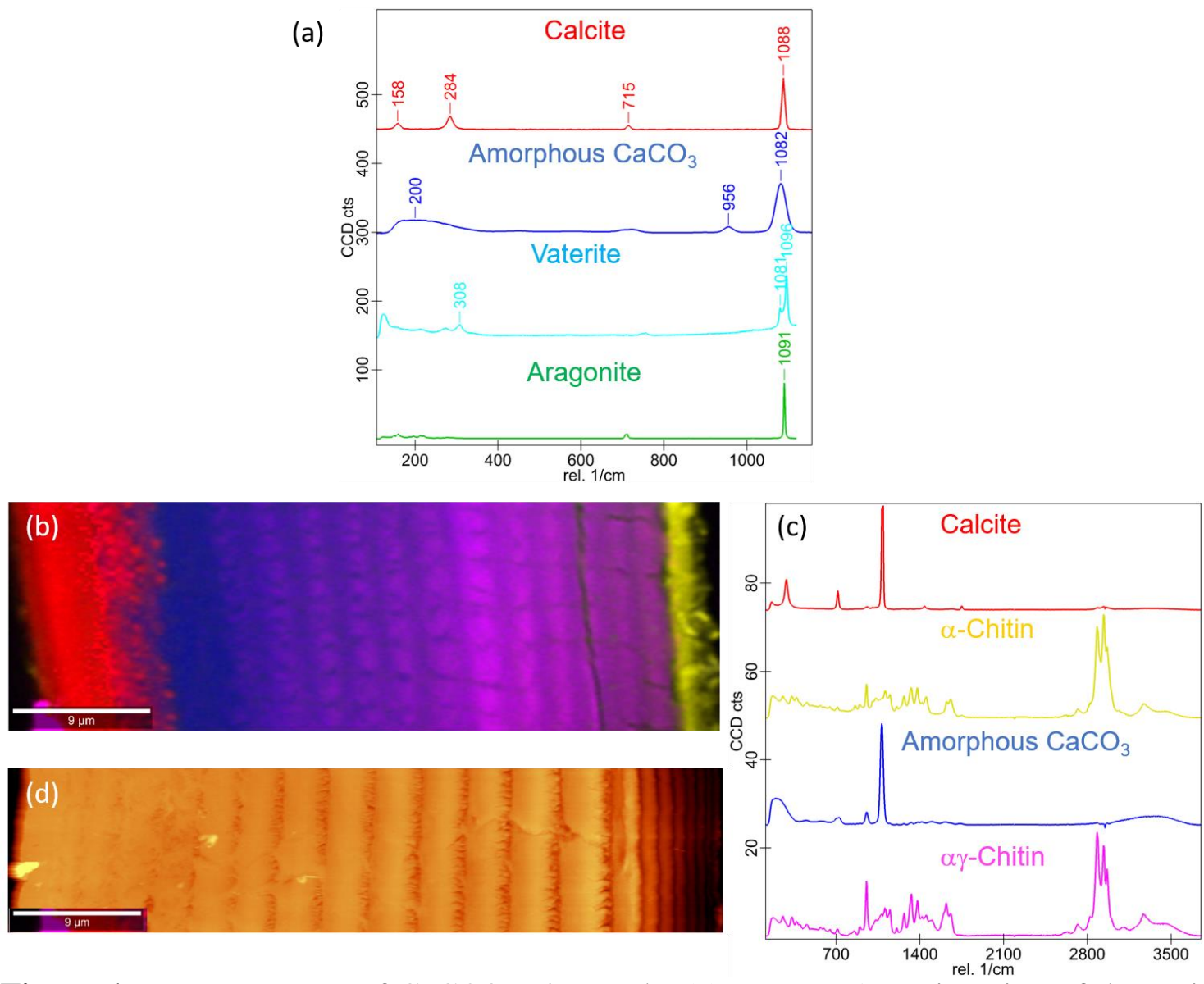

Figure 1. Raman spectra of $\mathrm{CaCO} 3$ polymorphs (a); Raman-AFM imaging of the cuticle of a porcellio scaber: color coded Raman image (b) with corresponding Raman spectra (c) and AFM image (d).

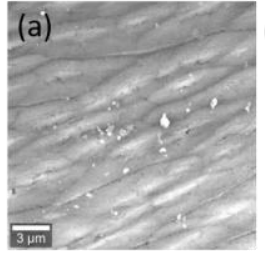

(b)

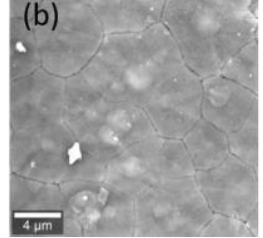

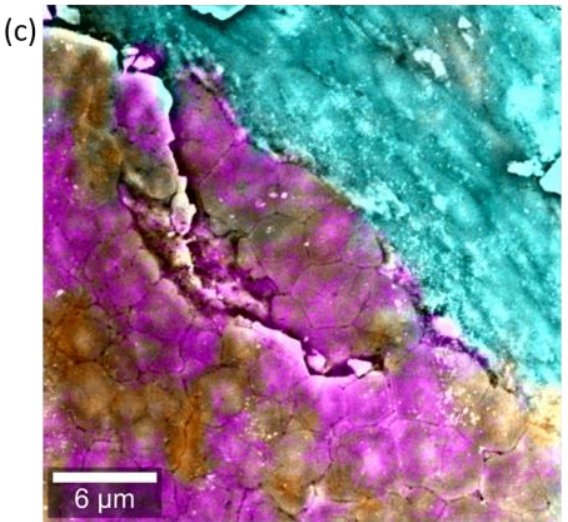

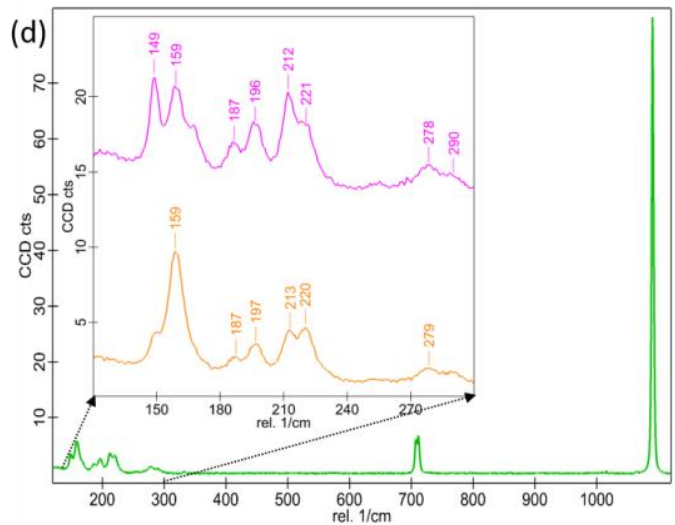

Figure 2. SEM images of vaterite (a) and aragonite (b); RISE image of the border between aragonite and vaterite (c) and Raman spectra of aragonite, highlighting the anisotropic behavior in the Ca lattice vibrations (d).

\section{References}

[1] H. Fabritius and A. Ziegler, J. Structural Biology 142 (2003) p. 281.

[2] U. Schmidt, S. Hild, A. Ziegler, Microscopy Today 17 (2009) p. 24.

[3] H. Francois-Saint-Cyr et al, Microscopy and Microanalysis. 22 (2016) p. 424.

[4] B. Pokroy et al, J. of Structural Biology 153 (2006) p. 145. 